\title{
LOOKING FOR ORDER IN THE OPTICAL DESIGN LANDSCAPE
}

\section{M. van Turnhout and F. Bociort}

Optics Research Group, Faculty of Applied Sciences, Delft University of Technology, The Netherlands E-mail: M.vanTurnhout@TUDelft.nl

\section{INTRODUCTION}

The presence of multiple local minima in the merit function landscape is one of the main difficulties in optical system design, especially for systems with many variables. By considering saddle points, we find a certain degree of order in the design landscape that alleviates the difficulty. We show a method to construct saddle points and easily obtain local minima from them. We make use of the fact that all local minima in the merit function space are connected via paths containing a certain type of saddle point.

\section{SADDLE-POINT CONSTRUCTION METHOD}

\section{Property of the design landscape}

- There is a relationship between local minima with a different number of variables.

- Many saddle points in the merit function landscape with $N$ variables result from local minima with $N-2$ variables (for simple systems about $70 \%$ ).

- We refer to this type of saddle as a "Null-element" Saddle Point (NESP).

\section{Practical design method for constructing NESPs}

Insert a meniscus lens with zero thickness and equal curvatures (a "nullelement") in a local minimum with $N-2$ variables.

\section{Mathematical idea}

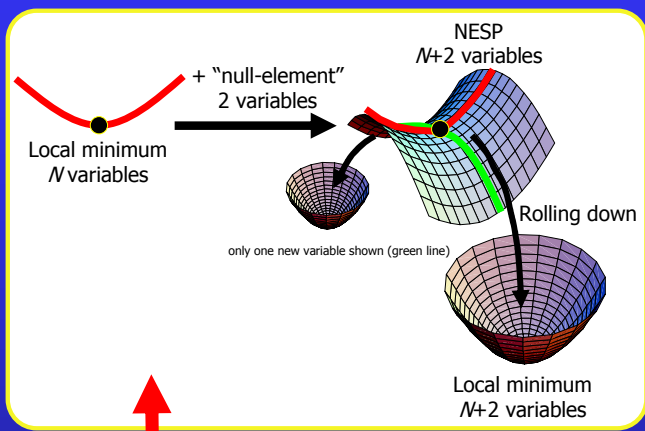

System shapes
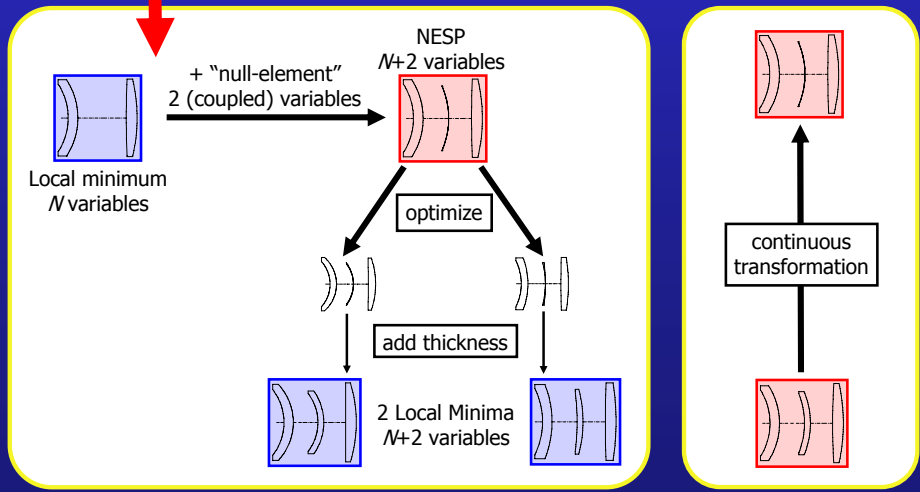

A NESP with thick lenses can be continuously transformed into a NESP which is constructed with our saddle-point construction method.
Computation of the "null-element" curvature

\section{Special case:}

- if the "null-element" glass = glass at reference surface $k$ (with curvature $c_{k}$ ),

- and if the "null-element" is inserted in contact with the reference surface $k$,

- then the "null-element" with surfaces having indices $k+1$ and $k+2$, respectively, has the property:

$$
c_{k+1}=c_{k+2}=c_{k}
$$

\section{General case:}

- insertion position of the "null-element" $\rightarrow$ arbitrary;

- the "null-element" glass $\rightarrow$ arbitrary;

- the "null-element" curvature $c_{s}$ can be calculated by using the fact that along the line

$$
c_{k+1}=c_{k+2}=c_{s},
$$

the merit function $f$ remains constant and that at a saddle point, the partial derivatives of $f$ must vanish:

$$
\begin{aligned}
& d f=\frac{\partial f}{\partial c_{k+1}} d c_{k+1}+\frac{\partial f}{\partial c_{k+2}} d c_{k+2}=0 \rightarrow \frac{\partial f}{\partial c_{k+1}}=-\frac{\partial f}{\partial c_{k+2}} \\
& \Rightarrow \frac{\partial f}{\partial c_{k+1}}=0 .
\end{aligned}
$$

It is a 1-dimensional numerical calculation!

Obtaining local minima

- Optimize downwards on both sides of the saddle:

$c_{k+1}=c_{k+2}=c_{s} \pm \varepsilon$,

where $\varepsilon$ indicates a small curvature change and $c_{s}$ is a solution of $\frac{\partial f}{\partial c_{k+1}}=0$.

$\rightarrow$ two distinct local minima.

- In the local minima, the zero distances between surfaces can be increased to the desired values.

- If the number of surfaces must be kept constant, then two surfaces (one lens) can be taken out of the resulting minima.

- By inserting and then extracting lenses new system shapes are obtained $^{1}$

Reference

1. O. Marinescu and F. Bociort, "Designing optics by using the order in the merit function space", Delft, The Netherlands, 2006

\section{CONCLUSIONS}

1. With saddle-point construction, many local minima can be obtained very rapidly from systems with fewer lenses, using a succession of 1-dimensional calculations.

2. In common practice, inserting a lens results in one local minimum. When a lens is inserted in a way that a saddle point is constructed, such a point leads after optimization to two local minima. For further design we can choose the best of them.

3. Thinking in terms of saddle points is still unfamiliar to most optical system designers. However, the results that can be expected justifies the effort for learning these new techniques and for integrating them in their work.

\section{http://www.optica.tn.tudelft.nl/users/bociort/networks.html}

